\title{
Associated Autoimmunity and Lead Exposure in Childhood
}

\author{
Deyanira Pacheco-Tovar ${ }^{\dagger}$, María-Guadalupe Pacheco-Tovar ${ }^{\dagger}$, Esperanza Avalos-Díaz, \\ Juan-José Bollain-y-Goytia, María-Elena Pérez-Pérez, Roxana Ramírez-Sandoval, \\ Argelia López-Luna, Rafael Herrera-Esparza*
}

Department of Immunology/ Academic Unit of Biological Sciences, Autonomous University of Zacatecas, Guadalupe, Zacatecas, Mexico

\section{Email address:}

qfbpato@hotmail.com (D. Pacheco-Tovar), pachecogpe@yahoo.com.mx (María-Guadalupe Pacheco-Tovar), avalosespera@gmail.com (E. Avalos-Díaz),jjbollainygoytia@uaz.edu.mx (Juan-José Bollain-y-Goytia), elepapperez@uaz.edu.mx (María-Elena Pérez-Pérez),rox_sandoval@hotmail.com (R. Ramírez-Sandoval), mariaa.lopez@hotmail.com (A. López-Luna),rafael.herreraesparza@gmail.com (R. Herrera-Esparza)

${ }^{*}$ Corresponding author

$\dagger$ Deyanira Pacheco-Tovar and María-Guadalupe Pacheco-Tovar are co-first authors.

\section{To cite this article:}

Deyanira Pacheco-Tovar, María-Guadalupe Pacheco-Tovar, Esperanza Avalos-Díaz, Juan-José Bollain-y-Goytia, María-Elena Pérez-Pérez, Roxana Ramírez-Sandoval, Argelia López-Luna, Rafael Herrera-Esparza. Associated Autoimmunity and Lead Exposure in Childhood. International Journal of Immunology. Vol. 9, No. 1, 2021, pp. 16-21. doi: 10.11648/j.iji.20210901.14

Received: February 28, 2021; Accepted: March 15, 2021; Published: March 26, 2021

\begin{abstract}
Background: The autoimmunity associated with environmental pollutants such as heavy metals is a phenomenon that has been described previously. The aim of the present study investigated the relationship between autoimmunity and lead pollutants in a cohort of children who lived in a mining settlement. Methods: Children were studied clinically and serologically. The serum lead concentration was measured by atomic absorption spectrometry, and antinuclear antibodies were analysed by immunofluorescence, ELISA and Western blot. Results: None of the studied children displayed any autoimmune disease. The lead concentration in the control group was negligible, at $0.10 \pm 011 \mu \mathrm{g}$. $\mathrm{L}^{-1}$. In contrast, the study group had values of $12.72 \pm 0.5 \mu \mathrm{g}$. $\mathrm{L}^{-1}$. The differences between the groups were significant $(\mathrm{p}<0.0001)$. In addition, control sera were negative for antinuclear antibodies (ANA), while the sera of children living near the mining settlement showed ANA-positive titres $(\mathrm{p}<0.001)$. These autoantibodies mainly recognized antigens associated with dividing cells, such as the mitotic apparatus and centrioles. Conclusion: The present results show how metallic pollutants induce environmentally associated autoimmunity and are reflected by antinuclear antibodies. This research should prompt us to take greater caution and implement permanent monitoring to ensure a healthy environment.
\end{abstract}

Keywords: Autoimmunity, Autoimmune Diseases, Autoantibodies, Antinuclear Antibodies, Enviromental Pollutants, Humoral Immunity

\section{Introduction}

Autoimmunity induced by heavy metals has been previously reported in animal models and in humans suffering from chronic metal ion intoxication. The effects on the immune system may result from the interaction between metal ions and normal proteins, which induce conformational changes in the proteins and trigger self-immune responses and/or cell hypersensitivity. Another way in which metals can stimulate the immune system is by inducing apoptosis and/or necrosis of the exposed tissues, with the consequent externalization of apoptotic remains, which may trigger an autoimmune response [1-4].

Human exposure to heavy metals is the result of anthropogenic industrial activities, especially mining and its related processes. Mining wastes contaminate soils, plants, water sources, and food; therefore, they may cause involuntary or accidental exposures in humans, and chronic exposure to heavy metals might elicit autoantibody production [5].

Zacatecas is a state located in north central México, and metallic ores are abundant in this area. A history of more than 
450 years of mining within the territory of Zacatecas has created a permanent risk of soil and water contamination near mining settlements. This is the result of certain ancestral techniques for silver extraction, which are no longer used. These environmental contaminants were mainly produced during the colonial period in the 17th century, polluting the soil with heavy metals, predominantly mercury and lead [6].

Previously, Manzanares-Acuña et al. [7] highlighted the presence of abnormal traces of lead in the blood of children who lived near mining areas in Zacatecas. This drew the attention of the state health department of Zacatecas (SSZ), which responded by adopting a permanent surveillance programme by monitoring lead traces in water sources, as well as lead testing in the blood of inhabitants of communities near mining areas.

The present investigation addresses two main questions, first, whether there is an increase in autoimmune rheumatic diseases in a cohort of children living near these mining sites, and second, whether there is any type of serologic manifestation of autoimmunity in these children.

To answer these questions, we tested the sera of a cohort of school-age children who were previously tested for lead contamination by the health authorities of the state and who were positive for elevated lead levels. The present study was focused on determining whether these children exhibited any clinical symptoms and/or antinuclear antibodies (ANAs) as a serologic manifestation of autoimmunity.

\section{Material and Methods}

\subsection{Studied Children}

A cohort of 48 school-age children were studied clinically and serologically. They lived in a mining municipality in the state of Zacatecas. The studied cohort consisted of 48 school children, of whom 24 attended an elementary school near mining facilities and 24 were from the same municipality but lived and attended school far from the mining site.

\subsection{Clinical Data Collection}

A complete clinical examination and directed questioning were performed to investigate systemic lupus erythematosus, rheumatoid arthritis, scleroderma, and juvenile inflammatory myopathies according to the internationally accepted criteria for the aforementioned diseases [8-11].

\subsection{Serum Sampling}

Specimens were obtained through the programme for lead contamination monitoring carried out in mining areas. Blood sampling was conducted according to the principles of the Helsinki Declaration [12], and the study was approved by the ethics committees of the participant institutions. In all cases, previous written informed consent was obtained from the parents of the studied children. Blood samples were collected by phlebotomy using the finger-prick capillary sampling procedure (Accu-Chek Softclix lancets, Roche), which was carried out according to the WHO guidelines for drawing blood [13]. The blood was collected in a polystyrene microtube, and after clotting, the microtubes were centrifuged in a refrigerated centrifuge at 2,000 rpm for 10 minutes. Serum samples were subjected to atomic absorption spectrometry (AAS) and antinuclear antibody determination by indirect immunofluorescence and Western blot assay.

\subsection{Atomic Absorption Spectrometry}

The lead levels in the sera were analysed by atomic absorption spectrometry in a Perkin Elmer (Norwalk, CT, USA) Analyst 300 atomic absorption spectrometer with an HGA 800 graphite furnace and an AS-72 autosampler. Chemicals were of analytical reagent grade. Serum samples were diluted $1: 4$ in $1 \% \mathrm{HNO}_{3}$, v/v and $0.02 \%$ cetyl trimethyl ammonium chloride (CTAC), the latter of which aids in metal extraction. Calibration was performed prior to the analysis with aqueous solutions in the range of $0.0-50.0 \mu \mathrm{g}$. $\mathrm{L}^{-1}$ lead $[14,15]$.

\subsection{Antinuclear Antibody Testing (ANA)}

Commercial slides containing HEp-2 cells (Immuno Concepts NA, Ltd, Sacramento, CA) were used for semiquantitative measurement based on the ANA titre starting at 1:40, 1:80, 1:160, 1:320, 1:640 and 1:1280. Serum dilutions were applied to HEp-2 cells and incubated for 30 minutes, followed by three PBS washes and a 30-minute incubation with FITC-labelled goat anti-human polyvalent gamma globulin (IgG, IgA, and IgM; Santa Cruz Biotechnology). The slides were washed in PBS and counterstained with DAPI (4',6-phenylindole diamidine-2' dihydrochloride, Roche). After 30 minutes, the slides were washed with methanol and methanol-PBS, mounted in fluorescence mounting medium (Dako, Cat. S3023, DakoCytomation) and then analysed at $20 \mathrm{X}$ and $40 \mathrm{X}$ under a fluorescence microscope (Olympus B-Max BX-40).

\subsection{Anti-dsDNA Antibodies}

Were assessed by immunofluorescence using Crithidia luciliae (Immuno Concepts NA, Ltd, Sacramento, CA), and slides were evaluated by fluorescence microscopy as previously described.

\subsection{Serum Specificity}

The recombinant proteins Ro60, La, and Sm/RNP (Euroimmun AG, Lubeck, Germany, and Orgentec Diagnostics GmbH, Mainz, Germany) were tested by ELISA, and the assays were carried out following the manufacturer's recommendations. Goat anti-human polyvalent HRP-labelled anti-IgG and IgM antibodies were used as secondary antibodies. Finally, the colour reaction was developed by incubating the sample for 30 minutes with the developer pNPP (p-nitro phenyl phosphate, disodium salt). The reaction was stopped, and the plates were assessed using an ELISA reader at $450 \mathrm{~nm}$. In all experiments, the five calibration solutions of the kit were used to construct the standard curve, and the results were expressed in optical density units. All 
assays were performed in triplicate.

\subsection{SDS-PAGE and Western Blot Analysis}

HEp-2 cell extracts were used as antigenic sources (HEp-2 cells ATCC ${ }^{\circledR}$ Catalog No. CCL-23. Protein quantification was performed by spectrophotometry using the Bradford method at $595 \mathrm{~nm}$ and UV extinction coefficient at $280 \mathrm{~nm}$. Cell extracts were separated on $10 \%$ SDS PAGE gels. Protein solutions were standardized to load $10 \mu \mathrm{L}$ of a $2 \mathrm{mg} / \mathrm{mL}$ final concentration of protein per well [16]. After electrophoresis, proteins were transferred onto nitrocellulose membranes (Hybond-C, Amersham, UK), and nonspecific binding of the antibodies was blocked with 5\% nonfat dry milk dissolved in PBS [17]. Immunoreactive bands were identified by applying a 1:1000 dilution of serum in $2 \%$ nonfat dry milk, $0.3 \%$ Tween $\AA 20$, PBS, and the membranes with diluted serum were incubated with gentle rocking overnight at $6^{\circ} \mathrm{C}$. Peroxidase-conjugated goat anti-human IgG (Santa Cruz Biotechnology) served as the secondary antibody. After a 1hour incubation, immunoreactive bands were detected by chemiluminescence according to the manufacturer's recommendations (Immun-StarTM Western C Chemiluminescence Kit, Bio-Rad), the protein band densities were determined in a Molecular Imager ChemiDoc XRS (Bio-Rad), and an antibody against $\beta$-actin was used as housekeeping protein.

\subsection{Statistical Analysis}

The data were processed using t-tests and linear regression in Prism software (GraphPad software). $\mathrm{p}<0.05$ was considered statistically significant.

\section{Results}

\subsection{Clinical Data}

The first group consisted of 24 children with an average age of $7.8 \pm 0.7$ years ( 9 females and 15 males). This group had previously tested positive for blood lead contamination since they lived in a community close to the mining settlement and was referenced as the study group. The 24 school-age children in the control group had an average age of $8.0 \pm 0.3$ years old, lived and attended primary school in an area distant from the mining site in the same municipality, and tested negative for blood lead.

In all cases, clinical history and complete physical examination were carried out by a physician accompanied by a nurse and in the presence of the child's relatives; a rheumatologist validated the clinical data. None of the children had clinical evidence of lupus, arthritis, myositis, scleroderma or other autoimmune diseases; after analysis, there were no significant differences in weight, height, psychomotor development or other clinical parameters between the two groups.

\subsection{Lead Blood Test}

To determine the degree of exposure, the total amount of serum lead was measured by the sensitive analytical technique of AAS, which is considered a useful tool for screening for diagnostic testing because it can detect amounts as low as $0.05 \mu \mathrm{g}$, which is considered in a very low detection limit range. The lead concentration level obtained in the control group was negligible, at $0.10 \pm 011 \mu \mathrm{g} . \mathrm{L}^{-1}$, in sharp contrast with the study group, which had abnormal lead levels of $12.72 \pm 0.5 \mu \mathrm{g}$. $\mathrm{L}^{-1}$. The difference between groups was significant at $\mathrm{p}<0.0001$ (Figure 1).

\section{Lead concentration}

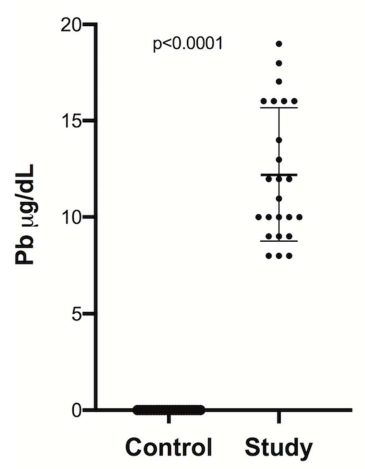

Antinuclear antibody

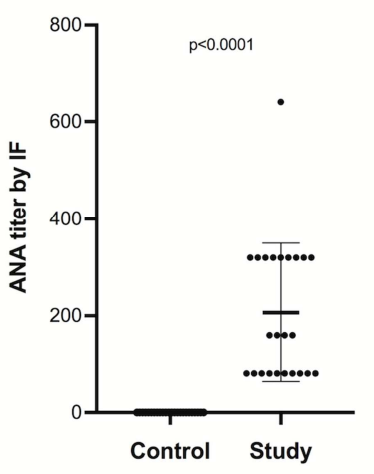

Lead/ANA

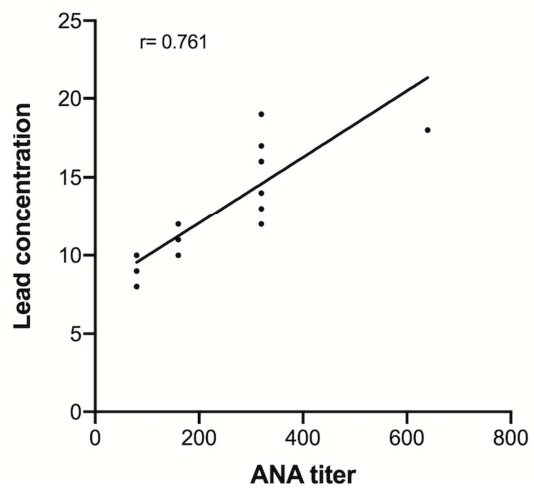

Figure 1. Antinuclear antibodies in children with elevated serum lead with a p value $<0.0001$. ANA positivity was observed only in the study group, and the difference from the control group was significant with a $p$ value $<0.0001$ (Student's $t$ test). At lower part of the graph, the relationship between lead concentration and ANA titre by immunofluorescence is shown. The correlation in this linear regression assay had a value of $r=0.761$.

\subsection{Antinuclear Antibody (ANA) Test}

Indirect immunofluorescence on HEp-2 cells is the gold standard for antinuclear antibody testing (ANA). This assay has been recently renamed anti-cell-antibody testing, and the fluorescent patterns are harmonized by a panel of experts.
The aforementioned criteria for standardization were used in our investigation. The unique difference was that the cut-off titre started at a 1:40 dilution [18]. In all the performed tests, control samples were included to compare the pattern specificity and fluorescence intensity [19]. None of the sera from the control group were ANA positive; however, the sera 
from the study group were ANA positive starting at the 1:80 dilution. Of these sera, fourteen samples had titres $1: 160$ or greater, and the difference between the groups was significant $(\mathrm{p}<0.001)$ (Figure 1). The results according to the guideline fluorescent patterns [20] were categorized as follows: $64 \%$ percent of sera exhibited a mitotic pattern (AC24 or centrosome in $32 \%$, and $\mathrm{AC}-26$ or NuMA in $32 \%$ ). Twenty percent had a nuclear pattern of $\mathrm{AC}-4$, or fine speckled, in $16 \%$ and AC-9, or nucleolar clumpy, in $4 \%$. The possible relationship between autoantibodies and lead concentration measured in $\mu \mathrm{g}$. $\mathrm{L}^{-1}$ (Y axis) and ANA titre (X axis) were estimated by a simple linear regression, which was significant with an $r$ value of 0.785 (Figure 1).

The specificity of ANA was studied by ELISA and immunofluorescence (Crithidia luciliae), and according to these assays, all sera were negative for anti-dsDNA, anti-Sm, anti-RNP, anti-Ro (SS-A) and anti-La (SS-B).

Additionally, we determined the molecular reactivity of ANA-positive sera by Western blot. The main results were as follows: $25 \%$ rather than $32 \%$ of the sera positive for the NuMA fluorescent pattern showed reactivity against a 234 $\mathrm{kDa}$ band corresponding to the NuMA protein. This band was identical to the band produced by an anti-NuMA prototype serum, and the remaining $7 \%$ of sera were negative for NuMA by Western blot. None of the control sera showed reactivity to this protein.

On the other hand, $29 \%$ of the sera with a centriole pattern showed reactivity against a $63 \mathrm{kDa}$ protein corresponding to alpha tubulin; this finding is probably nonspecific since $8 \%$ of the control sera were also reactive against tubulin. Interestingly, $8 \%$ of sera from the study group showed reactivity against a band of approximately $57 \mathrm{kDa}$ that, based on its immunolocalization by immunofluorescence, probably corresponds to Cep57-pericentrin protein. This protein is recruited by the pericentrin belt and is important for centriole organization, and none of the control group was positive for Cep57 [21] (Figure 2).
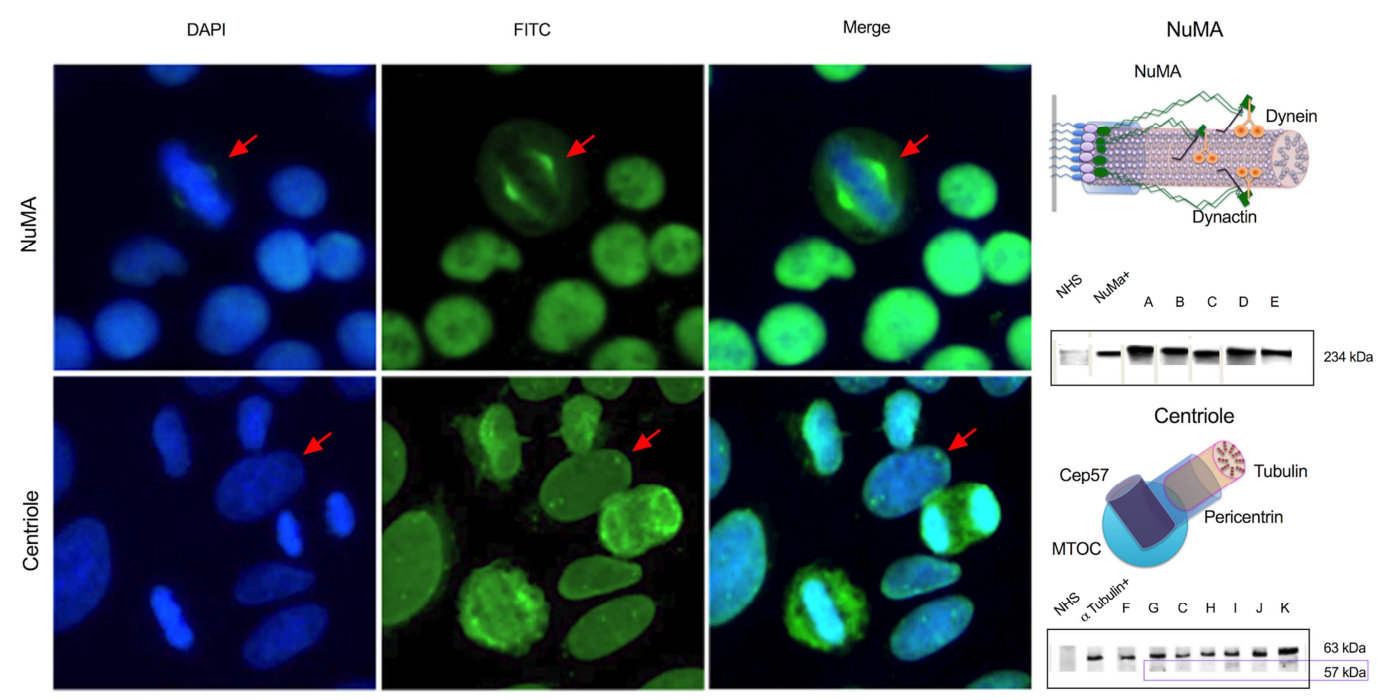

Figure 2. Indirect immunofluorescence showing dividing cells. The top panels show the NuMA pattern associated with a $234 \mathrm{kDa}$ band on Western blot (A to E); additionally, a schematic that shows how the NuMA protein is associated with microtubules and MTOC (Microtubule-organizing center) is given. The lower panels show the centriole pattern associated with a band of $63 \mathrm{kDa}$ that correspond to $\alpha$-Tubulin (F to K) and a band of $57 \mathrm{kDa}$, probably corresponding to Cep57-pericentrin (J, I and $K$ ), which helps to organize the centriole via the pericentrin complex. The schematic illustrates the association of Cep57-pericentrin with pericentrin and MTOC. Left, DAPI staining; Centre, reactivity of one serum sample from the study group; Right, merged image.

\section{Discussion}

The present investigation studied the relationship between autoimmunity and elevated serum lead in a cohort of children living in a mining settlement in Zacatecas, Mexico. The results of this cross-sectional survey did not show an increase in autoimmune diseases in children who were inadvertently contaminated with lead through environmental exposure. However, the presence of low-affinity antinuclear antibodies was detected; interestingly, these autoantibodies mainly recognized antigens of dividing cells, such as the mitotic apparatus and centrioles. This serologic finding was considered an autoimmune phenomenon rather than autoimmune disease, without clinical repercussions at the time that the study was carried out.
The autoimmunity associated with environmental pollutants such as heavy metals is a phenomenon that has been studied widely, and a strong body of epidemiological and experimental evidence suggests that exposure to mercury, lead, and other metals could be associated with the development of autoimmunity. Animal models and in vitro studies have contributed to a better understanding of the molecular mechanisms by which metallic pollutants can induce immune dysregulation [2].

The research in humans has limitations because the majority of reports are incidental, and there are no universally accepted criteria for the diagnosis or classification of environmentally associated autoimmunity. Nevertheless, most of the researchers in this area agree about the potential risk of autoimmunity due to chronic exposure to 
metals; therefore, age (children are more prone to inadvertent exposure with heavy metals), gender (females more than males), time of exposure (chronic more common than acute exposure), type of contaminating metal, and occupational and cultural factors, among other variables, are important in the development of autoimmunity [14, 22].

Human intoxication with heavy metals depends on various factors related to anthropogenic activities, such as the extractive processes of mining. There are some mining settlements in Mexico where the metallic extraction of silver started centuries ago, and these obsolete and polluting processes yielded an almost permanent environmental pollution with mercury and lead [6]. These mining settlements have not yet recovered because soil remediation technologies are expensive. Another problem is that some small communities and cities in Mexico were built centuries ago near mining boom sites and did not follow the official UNESCO recommendations, such as the establishment of environmental buffer zones; in other words, there is not a considerable distance between the cities and the mining settlements [23]. The historical environmental pollution has been perpetuated in the soil and water sources and may lead to human exposures, as in the case of the studied children who lived near a mining community.

Although it is not clear how metallic pollutants present in the environment induce autoimmunity, it is evident that the presence of lead in blood surely influences the B cell response against various autoantigens, as shown in the present study.

Experimental evidence of autoimmunity induced by xenobiotic mercury $\left(\mathrm{HgCl}_{2}\right)$ demonstrates that autoantibody production does not depend on the type I interferon response but rather depends on the signalling and trafficking of TLR7/9 in the late endosome or endolysosome that holds the UNC93B1/AP-3 complex [24]. Additionally, apoptosis and/or necrosis induced by heavy metals such as $\mathrm{Hg}, \mathrm{Au}$ and $\mathrm{Ag}$ and modification of influx $\mathrm{Ca}^{2+}$ channels may induce abnormal signalling that results in inflammation or autoimmunity [25]. Additionally, metallic pollutants such as lead may affect the hydrophobic centres of proteins or may disrupt disulfide bridges or salt bridges and modify secondary or tertiary structures. The effect of metals on proteins can be predicted by determining the sites of adhesion dependent on metal ions or MIDAS [2]. In sum, conformational changes in a protein could increase its antigenicity, favouring immunologic tolerance breakdown.

The association of traces of lead in the blood and the presence of antinuclear antibodies reported here suggests that this pollutant stimulated the production of antinuclear antibodies. Interestingly, the molecular reactivity of the aforementioned autoantibodies was fundamentally reactive against structures of dividing cells, and the main molecular targets were NuMA and the centriole, possibly including a molecule called Cep57-pericentrin that helps to organize the centriole via the pericentrin complex.

\section{Conclusion}

Present study demonstrated that children who were inadvertently contaminated with lead, developed antinuclear antibodies. We consider that beyond the analysis of specificity of antinuclear antibodies, our results constitute an example of how environmental pollutants are capable of inducing autoimmunity, and the findings of this research should prompt us to increase education for the remediation and permanent monitoring of water and soil and promote a healthy environment and better quality of life for human beings.

\section{Funding}

This work was supported by PROMEP-UAZ-CA-5 Autoinmunidad, Grant no. 103.5/09/1327 to Rafael HerreraEsparza.

\section{Competing Interests}

The authors declare that they have no competing interests.

\section{Acknowledgements}

We thank the Servicios de Salud de Zacatecas for allowing us to study the sera collected in their work.

\section{References}

[1] Ramírez-Sandoval R, Vázquez-del-Mercado M, DaneriNavarro A, López-Robles E, Bollain-y-Goytia JJ, Avalos-Díaz E, Herrera-Esparza R (2008) Intracellular antigens released from $\mathrm{Balb} / \mathrm{c}$ mice kidneys under chemically induced apoptosis and/or necrosis. Reumatismo 60: 108-113.

[2] Ramírez-Sandoval R, Luévano-Rodríguez N, RodríguezRodríguez M, Pérez-Pérez ME, Saldívar-Elias S, Gurrola-Carlos R, Avalos-Díaz E, Bollain-y-Goytia JJ, Herrera-Esparza R (2015) An animal model using metallic ions to produce autoimmune nephritis. J Immunol Res 2015: Article ID 269610.

[3] Pollard KM, Cauvi DM, Toomey CB, Hultman P and Kono DH (2019) Mercury-induced inflammation and autoimmunity. Biochim Biophys Acta Gen Subj 1863: 129299.

[4] Hudson W, Cao L, Kasten-Jolly J, Kirkwood JN, Lawrence DA (2003) Susceptibility of lupus-prone NZM mouse strains to lead exacerbation of systemic lupus erythematosus symptoms. J Toxicol Environ Health. Part A 23: 895-918.

[5] Gardner RM, Nyland JF, Silva IA, Ventura AM, De Souza JM, Silbergeld EK (2010) Mercury exposure, serum antinuclear/antinucleolar antibodies, and serum cytokine levels in mining populations in Amazonian Brazil: A crossSectional study. Enviromental Research 110: 345-354.

[6] González-Dávila O, Gómez-Bernal JM, Ruiz-Huerta EA (2012) Plants and soil contamination with heavy metals in agricultural areas of Guadalupe, Zacatecas, México. In: Environmental Contamination. Edited by Jatin Srivastava. Chap. 3. IntechOpen Ed, Rijtena, Croatia. pp 37-50. ISBN 978-953-51-0120-8. 
[7] Manzanares-Acuña E, Vega-Carrillo HR, Salas-Luévano MA, Hernández-Dávila VM, Letechipía-de-León C, BañuelosValenzuela R (2006) Lead levels in high-risk populations surrounding environment in San Ignacio, Fresnillo, Zacatecas, México. Salud Publica Mex 48: 212-219.

[8] Lundberg IE, Tjärnlund A, Bottai M, Werth VP, Pilkington C, Visser $M$ et al. (2017) European League Against Rheumatism/American College of Rheumatology classification criteria for adult and juvenile idiopathic inflammatory myopathies and their major subgroups. Arthritis Rheumatol 69: 2271-82, 2017.

[9] van den Hoogen F, Khanna D, Fransen J, Johnson SR, Baron M, Tyndall A et al (2013) 2013 Classification Criteria for Systemic Sclerosis. An American College of Rheumatology/European League Against Rheumatism Collaborative Initiative. Arthritis Rheum 65: 2737-47.

[10] Aringer M, Costenbader K, Daikh D, Brinks R, Mosca M, Ramsey-Goldman R et al (2019) 2019 European League Against Rheumatism/American College of Rheumatology Classification Criteria for Systemic Lupus Erythematosus. Arthritis Rheumatol 71: 1400-1412.

[11] Kay J and Upchurch KS (2012) ACR/EULAR 2010 Rheumatoid Arthritis Classification Criteria. Rheumatology (Oxford) 51(Suppl 6): vi5-9.

[12] World Health Organization (2001) World Medical Association Declaration of Helsinki. Ethical principles for medical research involving human subjects. Bull World Health Organ 79: 373-374.

[13] World Health Organization (2010) WHO guidelines on drawing blood: best practices in phlebotomy, Chapter 6 . Paediatric and neonatal blood sampling, pp 35-39, ISBN 978 924159922 1, Geneva, Switzerland.

[14] Abadin H, Ashizawa A, Stevens YW, Llados F, Diamond G, Sage G, Citra M, Quinones A, Bosch SJ, Swarts SG (2007) Toxicological profile for lead, U.S, Department of Health and Human Services. Agency for toxic substances and disease registry. Atlanta (GA). PMID: 24049859.

[15] Andrada D, Pinto FG, Gonçalves C, Nunes BR, Franco MB, Borba da Silva JB (2006) Direct determination of lead in human urine and serum samples by electrothermal atomic absorption spectrometry and permanent modifiers. J Braz Chem Soc 17: 328-332.

[16] Laemmli UK (1970) Cleavage of structural proteins during the assembly of the head of bacteriophage T4. Nature 227: 680685 .

[17] Towbin H, Staehelin T, Gordon J (1979) Electrophoretic transfer of proteins from polyacrylamide gels to nitrocellulose sheets: procedure and some applications. Proc Natl Acad Sci USA 76: 4350-4354.

[18] Rohwäder E, Locke M, Fraune J, Fechner K (2015) Diagnostic profile on IFA 40: HEp-20-10- an immunofluorescent test for reliable antinuclear antibody screening. Expert Rev Mol Diagn 15: 451-462.

[19] Dellavance A, Andrade LEC Detection of autoantibodies by direct immunofluorescence cytochemistry on HEp-2 cells. Methods Mol Biol 2019: 19-46, 2019.

[20] Damoiseaux J, Andrade LEC, Carballo OG, Conrad K, Francescantonio PLC, Fritzler MJ et al. (2019) Clinical relevance of HEp-2 indirect immunofluorescent patterns: the International Consensus on ANA (ICAP) perspective. Ann Rheum Dis 78: 879-889.

[21] Watanabe K, Takao D, Ito KK, Takahashi M, Kitagawa D (2019) The Cep57-pericentrin module organizes PCM expansion and centriole engagement. Nat Commun 10: 931.

[22] Pollard KM (2015) Environment, autoantibodies, and autoimmunity. Front Immunol 6: 60 eCollection 2015.

[23] Kholodov A, K. Golokhvast K (2020) Air pollution of nature reserves near cities in Russia. Scientifica (Cairo) Article ID 9148416. eCollection 2020.

[24] Pollard KM, Escalante GM, Huang H, Haraldsson K M, Hultman P, Christy JM, Pawar RD, Mayeux JM, GonzalezQuintial R, Baccala R, Beutler B, Theofilopoulos AN, Kono DH (2017) Induction of systemic autoimmunity by xenobiotic requires endosomal TLR trafficking and signaling from the late endosome/endolysosome but not type-I IFN. J Immunol 199: 3739-3747.

[25] Suzuki Y, Inoue T, Ra C (2011) Autoimmunity-inducing metals ( $\mathrm{Hg}, \mathrm{Au}$ and $\mathrm{Ag}$ ) modulate mast cell signaling, function and survival: Curr Pharm Des 17: 3805-3814. 